\title{
Psychosocial profile of children and adolescents followed in a pediatric musculoskeletal pain clinic
}

\author{
Vânia Schinzel, Juliana Molina, Melissa Fraga, Vanessa Miotto e Silva, Maria Teresa Terreri, Claudio A Len \\ From 21st European Pediatric Rheumatology (PReS) Congress \\ Belgrade, Serbia. 17-21 September 2014
}

\begin{abstract}
Introduction
Amplified pain syndrome preferentially affects girls between the ages of 10-17 years. It is a disease with multiple causes that could be associated with major psychosocial disorders of patients and caregivers, affecting their quality of life. These psychosocial aspects can interfere intensifying the pain.
\end{abstract}

\section{Objectives}

This is a transversal study with the objective of evaluating the quality of life of patients seen in a pediatric musculoskeletal pain clinic, drawing a panorama of the educational, social, and psychological aspects.

\section{Methods}

25 patients from our Pediatric Musculoskeletal Pain Clinic were consecutively selected. The patients and their caregivers responded to the following questionnaires: Children's Depression Inventory (CDI), PedsQL ${ }^{\mathrm{TM}}$ (Pediatric Quality of Life Inventory ${ }^{\mathrm{TM}}$ ) 4.0, PedsQL Multidimensional Fatigue Scale, family APGAR score, and SF-36 Health Survey.

\section{Results}

We included 25 patients between the ages of 8 and 17 , with an average of 12.6 years, $68 \%$ girls. In relation to the CDI, $95 \%$ were below the cutoff point, average $=5.72$. The highest score was 18 points. In the Family APGAR, the average was 13.4. In the PedsQL 4.0 the score of patients were between 20.8 and 95.1 with an average $=62.6$ and $\mathrm{SD}=19.8$. The score from the point of views of patients were between 25.15 and 90.1, with an average-59.5 and $\mathrm{SD}=18.6$. Regarding the PedsQL-Fatigue, the variation was between 20.8 and 91.7, with an average $=60.9$ and $\mathrm{SD}=17.4$, and the caretakers varied between 18.0 and 93.0 with an average $=60.6$ and $\mathrm{SD}=22.3$. In the $\mathrm{SF}-36$ the patients obtained averages (SD) of $57.7(29.14) ; 43.2$ (47.14); 46.5(21.87); 47.4(20.88); 52.2(19.16); 54.4(28.46); 56.3(35.36), and 61.9(24.30) for the domains: Functional capacity, limitation from physical aspects, pain, general health, vitality, social aspects, emotional and mental health aspects, respectively.

\section{Conclusion}

The patients did not present high rates of depression. However, we observed problems in family relationship and social life. Psychological problems could establish a causal relationship in some cases or reinforce the sensation of pain.

\section{Disclosure of interest}

None declared.

Published: 17 September 2014

doi:10.1186/1546-0096-12-S1-P104

Cite this article as: Schinzel et al.: Psychosocial profile of children and adolescents followed in a pediatric musculoskeletal pain clinic. Pediatric Rheumatology 2014 12(Suppl 1):P104. 